\title{
Rituximab en lupus sistémico ampolloso
}

Mariana Cruz, Diego Carlos Graña-Cruz, Leticia Pérez, Javier Gaudiano

Servicio de Clínica Médica. Hospital Pasteur. Universidad de la República. Montevideo, Uruguay

Recibido: 03/08/2020

Aceptado: 22/10/2020

En línea: 31/12/2020

Citar como: Cruz M, Graña-Cruz DC, Pérez L, Gaudiano J. Rituximab en lupus sistémico ampolloso. Rev Esp Casos Clin Med Intern (RECCMI). 2020 (Dic); 5 (3): $133-136$. doi: $10.32818 /$ reccmi.a5n3a8.

Cite this as: Cruz M, Graña-Cruz DC, Pérez L, Gaudiano J. Rituximab in Refractory Cutaneous Lupus. Rev Esp Casos Clin Med Intern (RECCMI). 2020 (Dec); $5(3)$ : $133-136$. doi: 10.32818/reccmi.a5n3a8.

Autor para correspondencia: Diego Carlos Graña-Cruz. diegograna@adinet.com.uy

\section{Palabras clave}

$\triangleright$ Lupus

$\triangleright$ Compromiso cutáneo

$\triangleright$ Rituximab

\section{Keywords}

$\triangleright$ Lupus

$\triangleright$ Skin involment

$\triangleright$ Rituximab

\section{Resumen}

El lupus eritematoso sistémico es una enfermedad crónica autoinmune de compromiso multiorgánico, infrecuente en el sexo masculino, donde se presenta con una mayor morbimortalidad. Se presenta el caso clínico de un hombre que debuta con compromiso sistémico grave, agregando compromiso cutáneo bulloso refractario a tratamiento inmunosupresor, con una buena respuesta posterior a rituximab.

\section{Puntos destacados}

$\triangleright$ Rituximab puede dar buenos resultados en algunos pacientes con lupus eritematoso cutáneo refractario.

\section{Introducción}

El lupus eritematoso sistémico (LES) es el prototipo de enfermedad autoinmune con afectación multiorgánica ${ }^{1,2}$.

El compromiso cutáneo ocurre en el 70\% de los casos. Las lesiones cutáneas pueden ser especificas o inespecíficas. Entre las primeras están el lupus eritematoso cutáneo (LEC) crónico, subagudo y agudo ${ }^{3,4}$. Entre las inespecíficas está el LES bulloso, enfermedad rara subepidérmica ${ }^{3,4}$.

\section{Caso clínico}

\section{Antecedente y exploración física}

Varón de 19 años que consulta por lesiones eritematosas pruriginosas a nivel de ambos miembros superiores acompañada de vesículas.
El examen físico mostraba placas de aspecto herpetiforme, descamantes a nivel de manos y antebrazos, algunas de ellas con lesiones ampollares, respetando palmas (Figuras 1, 2 y 3); sin eritema malar, ulceras orales, ni otros elementos de compromiso cutáneo. Edema de cara periorbitario y orinas espumosas.

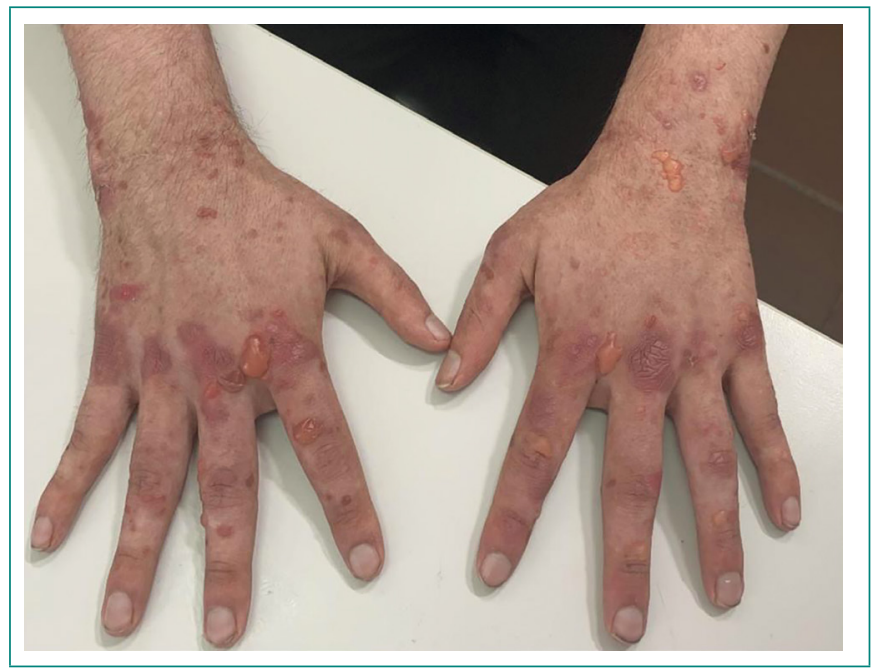

Figura 1. Placas urticariformes a nivel de manos y antebrazos, algunas de ellas con lesiones ampollares. 


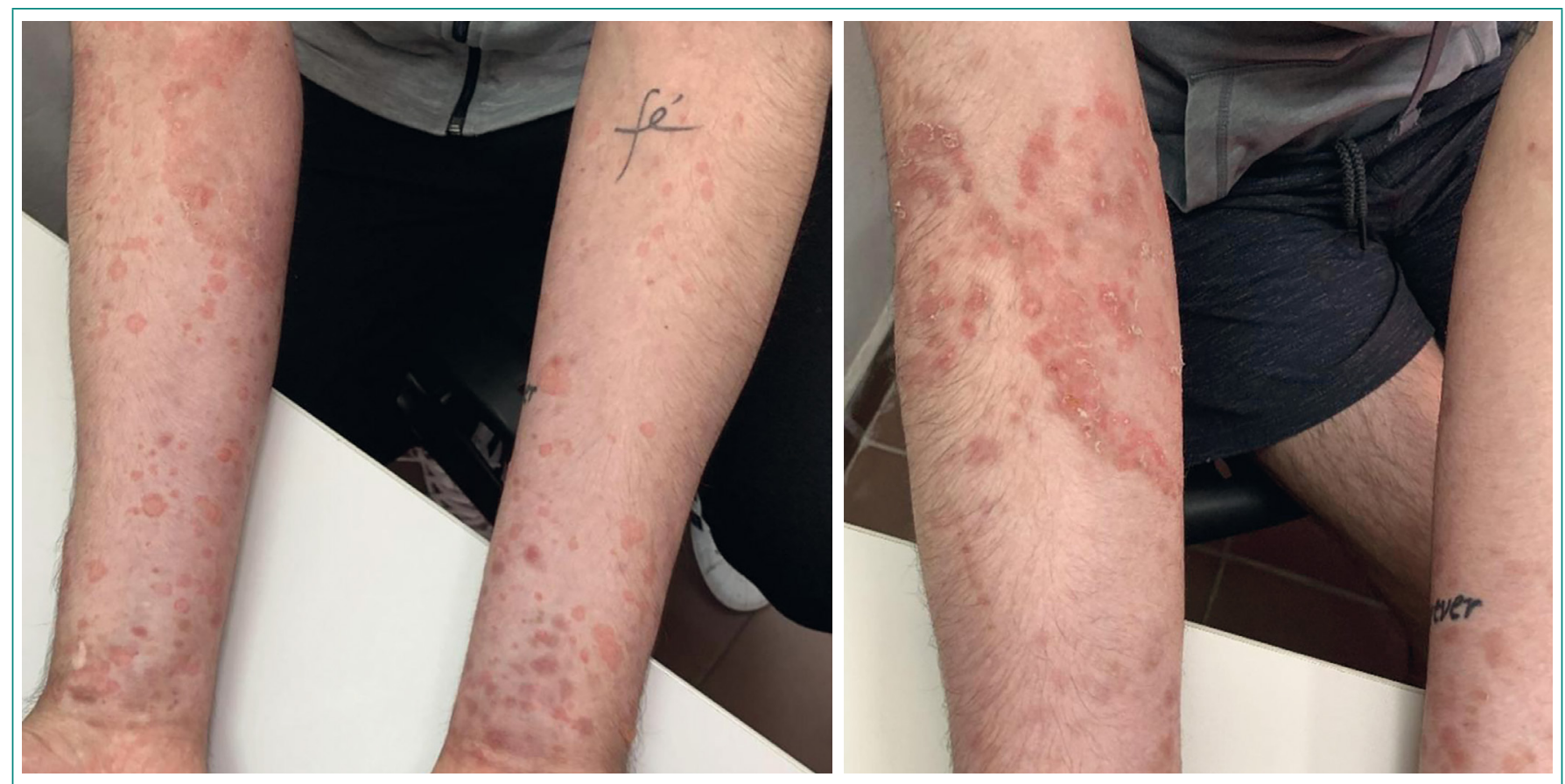

Figuras 2 y 3 . Lesiones ampollares con características urticariformes en ambos antebrazos.

\section{Pruebas complementarias}

Se realizan las siguientes pruebas complementarias:

- Hb 10,2 g/dL, VCM 83 fl, HCM 26 pg, plaquetas 109.000 mm³, leucocitos: $2.530 \mathrm{~mm} 3$; linfocitos $1.040 \mathrm{~mm}^{3}$.

- Proteína C reactiva de 2 .

- Creatinina de 1,14 mg/dL, azoemia 0,67 g/L.

- Albumina 2,1 g/dL.

- Examen de orina: proteinuria 6,6 g/L, índice proteininuria/creatininuria 3,8.

- Glóbulos rojos dismórficos.

- Anticuerpos antinucleares (ANA) 1/1280 patrón homogéneo; Anti SM + / Anti RNP + / Anti Ro +/ Anti LA +; Anti DNA +.

- C3 0,2 mg/dL y C4 0,0 mg/dL; velocidad de eritrosedimentación de $125 \mathrm{~mm} / \mathrm{h}$.

- Test de Coombs positivo.

Los hallazgos son compatibles con un lupus eritematoso sistémico con compromiso cutáneo, renal y hematológico cumpliendo los criterios clasificatorios establecido por SLICC/ACR 2013. Se plantea una punción biópsica renal con el resultado de nefropatía lúpica tipo IV, SLEDAI de $16^{5}$ (Tabla 1).

Se inicia bolo de metilprednisolona (MPD) $1 \mathrm{~g} /$ día durante tres días, pauta de corticoides descendente, micofenolato $3 \mathrm{~g}$ día e hidroxicloroquina $200 \mathrm{mg} /$ día. Al cabo de un mes del inicio del tratamiento, se observa mejoría de la afectación renal y hematológica, pero agravamiento del compromiso cutáneo persistiendo con un SLEDAI de 10.

Se plantea compromiso cutáneo refractario. Se indica nueva serie de bolos de MPD + ciclofosfamida (CFM) con mala respuesta, observándose al tercer bolo de CFM, extensión de las lesiones bajo tratamiento. Se indica rituximab $1 \mathrm{gr}$ IV en 2 tomas cada 15 días. A los seis meses del inicio del tratamiento, presenta mejoría franca del compromiso cutáneo manteniendo la remisión a nivel renal y hematológico con un SLEDAI de 2 .

\section{Diagnóstico}

Lupus sistémico ampolloso refractario.

\section{Discusión}

EI LEC es un hallazgo frecuente en los pacientes con LES, pudiendo alcanzar un $70 \%$ de las manifestaciones. Constituye muchas veces su carta de presentación $y$, en algunos casos, se presenta aún en ausencia de enfermedad sistemica ${ }^{6,7}$.

El lupus cutáneo bulloso es una forma de presentación atípica, que suele aparecer en menos del $5 \%$ de los pacientes, asociado a una gran actividad y, sobre todo, a la presencia de nefritis lupica ${ }^{8}$. Si bien es más frecuente en mujeres de raza negra, es compatible con nuestro caso, por la gran actividad sistémica y un compromiso renal proliferativo ${ }^{4}$. Histológicamente se comporta de forma muy similar al pénfigo bulloso y la dermatitis herpetiforme.

Las lesiones pueden aparecer en cualquier sector. Frecuentemente se observan en tronco, cuello y miembros superiores, como en nuestro caso clínico, observándose cierta tendencia a presentarse en aquellas áreas fotoexpuestas ${ }^{4,9}$.

Su tratamiento constituye un desafío, existiendo pocos estudios comparativos al respecto.

Aunque la fotoprotección, el tratamiento tópico y sistémico con corticoides y antipalúdicos se consideran un tratamiento de primera línea, un 50\% de los pacientes se comportan de forma refractaria ${ }^{10,11}$. En estos pacientes las drogas inmunosupresoras, como azatioprina, metotrexato, micofenolato de mofetilo y ciclosporina, pueden constituir un tratamiento de segunda línea. Sin embargo muchos pacientes suelen ser refractarios a estas drogas ${ }^{10,11}$. Si bien está descrito el uso de dapsona y tacrolimus en pacientes con lupus cutáneo refractario, no son de fácil acceso en nuestro medio y no contamos con experiencia suficiente para el uso de estos fármacos.

En un estudio realizado en 2017 publicado por la revista JAMA Dermatology, dentro de los fármacos no-antimaláricos, la talidomida y el metrotexate fueron la opción más efectiva ${ }^{10}$. Aunque la talidomida tiene un alto riesgo de efectos adversos, constituye la terapia con una respuesta mayor ${ }^{10}$. No consideramos su utilización en nuestro paciente por la dificultad de acceso así como por sus potenciales efectos adversos. 


\begin{tabular}{|c|c|c|c|}
\hline Puntuación & SLEDAI & Descriptor & Definición \\
\hline 8 & & Convulsiones & De comienzo reciente. Excluir causas infecciosas, metabólicas y fármacos. \\
\hline 8 & & Psicosis & $\begin{array}{l}\text { Habilidad alterada para la función diaria debido a alteración grave en la percepción de la realidad. } \\
\text { Incluye alucinaciones, incoherencia, asociaciones ilógicas, contenido mental escaso, pensamiento } \\
\text { ilógico, raro, desorganizado y comportamiento catatónico. Excluir l. renal y fármacos. }\end{array}$ \\
\hline 8 & & $\begin{array}{l}\text { Sdme orgánico- } \\
\text { cerebral }\end{array}$ & $\begin{array}{l}\text { Función mental alterada con falta de orientación, memoria, u otras funciones intelectuales, } \\
\text { de comienzo rápido y manifestaciones clínicas fluctuantes. Incluye disminución del nivel de } \\
\text { conciencia con capacidad reducida para focalizar, e inhabilidad para mantener la atención en el } \\
\text { medio, más, al menos dos de los siguientes: alteración de la percepción, lenguaje incoherente, } \\
\text { insomnio o mareo matutino, o actividad psicomotora aumentada o disminuida. Excluir causas } \\
\text { infecciosas, metabólicas y fármacos. }\end{array}$ \\
\hline 8 & & Alteraciones visuales & $\begin{array}{l}\text { Retinopatía lúpica. Incluye cuerpos citoides, hemorragias retinianas, exudados serosos y } \\
\text { hemorragias en la coroides, o neuritis óptica. } \\
\text { Excluir HTA, infección o fármacos. }\end{array}$ \\
\hline 8 & & Cefalea lúpica & Grave, persistente; puede ser migrañosa pero no responde a analgésicos narcóticos. \\
\hline 8 & & AVC & De reciente comienzo. Excluir arteriosclerosis. \\
\hline 8 & & Vasculitis & $\begin{array}{l}\text { Ulceración, gangrena, nódulos dolorosos sensibles, infartos periungueales, hemorragias en astilla o } \\
\text { biopsia o angiografía que confirme la vasculitis. }\end{array}$ \\
\hline 4 & & Miositis & $\begin{array}{l}\text { Debilidad proximal/dolor asociado a elevación de las CPKValdolasa o EMG sugestivo o miositis } \\
\text { comprobada por biopsia. }\end{array}$ \\
\hline 4 & & Artritis & Más de dos articulaciones dolorosas y con signos inflamatorios. \\
\hline 4 & & Cilindros urinarios & Cilindros hemáticos o granulosos. \\
\hline 4 & & Hematuria & > 5 hematíes/c. Excluir litiasis, infección u otras causas. \\
\hline 4 & & Proteinuria & $>5$ g/24 h. De reciente comienzo o aumento de la proteinuria ya conocida en más de 0,5 g/24 h. \\
\hline 2 & & Exantema nuevo & Comienzo reciente o recurrente. Exantema inflamatorio. \\
\hline 2 & & Alopecia & De comienzo reciente o recurrente. Pérdida difusa o en placas. \\
\hline 2 & & Ulceras bucales & De comienzo reciente o recurrente. Ulceras bucales o nasales. \\
\hline 2 & & Pleuritis & Dolor pleurítico con roce o derrame, o engrosamiento pleural. \\
\hline 2 & & Pericarditis & $\begin{array}{l}\text { Dolor pericárdico con al menos uno de los siguientes: roce, derrame, cambios electrocardiográficos } \\
\text { o confirmación ecocardiográfica. }\end{array}$ \\
\hline 2 & & Complemento & Descenso de CH50, C3, C4 por debajo del límite inferior del laboratorio. \\
\hline 2 & & Anti DNA & $>25 \%$. Técnica de Farr o por encima del valor habitual del laboratorio. \\
\hline 1 & & Fiebre & $>38^{\circ} \mathrm{C}$. Excluir infección. \\
\hline 1 & & Trombopenia & $<100.000$ plaquetas $/ \mathrm{mm}^{3}$ \\
\hline 1 & & Leucopenia & $<3.000$ células $/ \mathrm{mm}^{3}$. Excluir fármacos. \\
\hline $\begin{array}{l}\text { PUNTUACION } \\
\text { TOTAL }\end{array}$ & & \multicolumn{2}{|c|}{ Nota: puntúa en la escala SLEDAl si el descriptor está presente en el día de la visita o 10 días antes. } \\
\hline
\end{tabular}

Tabla 1. Índice de actividad del lupus eritematoso sistemico. SLEDAI (Systemic Lupus Erythematosus Disease Activity Index, Bombardier et al., 1992).

El rituximab, anticuerpo monoclonal quimérico dirigido contra el antígeno CD20, se ha utilizado en los casos refractarios, con buenos resultados en lupus bulloso; sin embargo, también se han descrito casos clínicos con refractariedad $^{9,11-15}$.

\section{Conclusiones}

El rituximab constituye una alternativa terapéutica válida para el tratamiento de los pacientes con LEC refractario a tratamientos convencionales. Es necesa- ria la realización de estudios comparativos con un mayor número de pacientes para poder extraer conclusiones significativas dado que la mayoría de la evidencia surge de casos clínicos y serie de casos.

\section{Bibliografía}

1. Boodhoo KD, Liu S, Zuo X. Impact of sex disparities on the clinical manifestations in patients with systemic lupus erythematosus: A systematic review and meta-analysis. Medicine (Baltimore). 2016; 95(29): e4272. doi: 10.1097/ MD.0000000000004272. 
2. Crow MK. Etiology and Pathogenesis of Systemic Lupus Erythematosus, Tenth Edit, Elsevier Inc, 2017. doi:10.1016/B978-0-323-31696-5.00079-6.

3. Sánchez-Navarro LM, Poletti ED, López-Domínguez NV, López SS, SánchezDelgado MA. Lupus eritematoso sistémico ampolloso: reporte de un caso y revisión de la literatura. DermatologíaCMQ. 2012; 10(3): 182-187. (Ultimo acceso Agosto 2020) Accesible en: https://www.medigraphic.com/pdfs/ cosmetica/dcm-2012/dcm123e.pdf

4. Sebaratnam DF, Murrell DF. Bullous systemic lupus erythematosus. Dermatol Clin. 2011; 29(4): 649-53. doi: 10.1016/j.det.2011.06.002.

5. Bombardier C, Gladman DD, Urowitz MB, Caron D, Chang CH. Derivation of the SLEDAI. A disease activity index for lupus patients. The Committee on Prognosis Studies in SLE. Arthritis Rheum. 1992; 35(6): 630-40. doi: 10.1002 art.1780350606

6. Okon LG,WerthVP.Cutaneous lupus erythematosus: diagnosis and treatment. Best Pract Res Clin Rheumatol. 2013; 27(3): 391-404. doi: 10.1016/j.berh.2013.07.008.

7. Stannard JN, Kahlenberg JM. Cutaneous lupus erythematosus: updates on pathogenesis and associations with systemic lupus. Curr Opin Rheumatol. 2016; 28(5): 453-459. doi:10.1097/BOR.0000000000000308.

8. Vico-Alonso C, Calleja-Algarra A, Andrés-Lencina JJ, Aragón-Miguel R, Pinilla-Martin B, Sánchez-Velázquez A, et al. Bullous eruption in a 1-year-old female in the Philippines: A diagnostic challenge. (Poster in American Academy of Dermatology 2019 Annual Meeting). J Am Acad Dermatol. 2017; 81(4) suppl.1: AB177. doi: 10.1016/j.jaad.2019.06.660.

9. de Risi-Pugliese T, Cohen Aubart F, Haroche J, Moguelet P, GrootenboerMignot S, Mathian A, et al. Clinical, histological, immunological presenta- tions and outcomes of bullous systemic lupus erythematosus: 10 New cases and a literature review of 118 cases. Semin Arthritis Rheum. 2018; 48(1): 83-89. doi: 10.1016/j.semarthrit.2017.11.003.

10. Fruchter R, Kurtzman DJB, Patel M, Merola J, Franks AG Jr, Vleugels RA, et al. Characteristics and Alternative Treatment Outcomes of AntimalarialRefractory Cutaneous Lupus Erythematosus. JAMA Dermatol. 2017; 153(9): 937-939. doi: 10.1001/jamadermatol.2017.1160

11. Penha MÁ, Libório RDS, Miot HA. Rituximab in the treatment of extensive and refractory subacute cutaneous lupus erythematosus. An Bras Dermatol. 2018; 93(3): 467-469. doi: 10.1590/abd18064841.20187561

12. Alsanafi S, Kovarik C, Mermelstein AL, Werth VP. Rituximab in the treatment of bullous systemic lupus erythematosus. J Clin Rheumatol. 2011; 17(3): 142-4. doi: 10.1097/RHU.0b013e318214f30c

13. Akpabio AA, Otike-Odibi BI. Severe bullous systemic lupus erythematosus successfully treated with low dose rituximab: a case report from subSaharan Africa. Reumatismo. 2020 Jul 23;72(2):115-119. doi: 10.4081/ reumatismo.2020.1274

14. Cieza-Díaz DE, Avilés-Izquierdo JA, Ceballos-Rodríguez C, Suárez-Fernández R. Lupus eritematoso cutáneo subagudo refractario tratado con rituximab [Refractory subacute cutaneous lupus erythematosus treated with rituximab]. Actas Dermosifiliogr. 2012 Jul-Aug;103(6):555-7. doi: 10.1016/j. ad.2011.10.013

15. Lowe CD, Brahe CA, Green B, Lam TK, Meyerle JH. Bullous systemic lupus erythematosus successfully treated with rituximab. Cutis. 2019; 103(6): E5-E7. 